\title{
EVENTOS TROMBOEMBÓLICOS EM MULHERES GRÁVIDAS E PUÉRPERAS
}

\author{
Aline Sharlon Maciel Batista Ramos ${ }^{1}$, Débora Carvalho Barros ${ }^{2}$, Hariane Freitas \\ Rocha Almeida ${ }^{3}$, Isabela Bastos Jácome de Souza ${ }^{4}$, Rafael Mondego Fontenele ${ }^{5}$. \\ 1'Doutoranda em Ciências da Saúde (UERJ). Docente Assistente da Universidade \\ CEUMA, São Luís - MA, Brasil. E-mail: alinesharlon@gmail.com \\ ${ }^{2}$ Graduada em Enfermagem (UniCEUMA). \\ ${ }^{3}$ Graduada em Enfermagem (UniCEUMA). Pós-graduanda em Auditoria, \\ Planejamento e Gestão em Saúde (FGB) e em Enfermagem do Trabalho (UCAM). \\ ${ }^{4}$ Graduada em Enfermagem (CEST). Mestre em Saúde da Família (UNINOVAFAPI). \\ Docente Assistente da Universidade CEUMA. \\ ${ }^{5}$ Graduado em Enfermagem (UniCEUMA). Mestre em Gestão de Programas e \\ Serviços de Saúde (UniCeuma). Docente da Faculdade Gianna Beretta.
}

\section{Recebido em: 22/09/2018 - Aprovado em: 23/11/2018 - Publicado em: 03/12/2018 DOI: 10.18677/EnciBio 2018B96}

\begin{abstract}
RESUMO
O tromboembolismo venoso (TEV) é uma das principais causas de morbimortalidade obstétrica nas mulheres que estão no ciclo gravídico-puerperal, pois a hipercoagulabilidade sanguínea, proporcionada na gravidez, aumenta significativamente a probabilidade do aparecimento dessa doença que está associada a alguns fatores de risco, muitas vezes desconhecidos. O objetivo desse estudo foi identificar os fatores de risco associados aos eventos tromboembólicos em mulheres no ciclo gravídico-puerperal. Foi realizada uma revisão de literatura utilizando as bases de dados eletrônicas PUBMED (Public Medline) e SCIELO (Scientific Eletronic Library Online). Para o levantamento dos estudos e ampliação das buscas foram utilizados os seguintes descritores: em português - Fatores de Risco; Gestação; Gravidez; Puerpério; Tromboembolismo Venoso, em inglês- Risk Factors; Gestation; Pregnancy; Puerperium; Venous Thromboembolism. A seleção de amostra obedeceu aos seguintes critérios de inclusão: artigos completos disponíveis eletronicamente; estar escrito no idioma português; estudos publicados no recorde temporal entre 2006 a 2016. Foram identificados, nas amostras dos estudos selecionados, 30 fatores de risco relacionados aos eventos tromboembólicos, sendo que sete desses fatores foram evidenciados mais de uma vez nos estudos, sendo eles: Idade, tabagismo, obesidade, trombofilia, cardiopatia, terceiro trimestre e repouso prolongado. Dentre os fatores de risco mais frequentes que levam mulheres grávidas e puérperas a sofrerem eventos tromboembólicos, podem-se destacar a obesidade e o fator idade maior que 35 anos como condições significativas; sendo a idade um fator de risco tanto para antenatal como puerperal. Fatores como tabagismo, trombofilia, cardiopatias, terceiro trimestre e repouso prolongado se apresentaram como igualmente relevantes para a ocorrência de tromboembolismo venoso.
\end{abstract}

PALAVRAS-CHAVE: Fatores de Risco. Gestação. Gravidez. Puerpério. Tromboembolismo Venoso. 


\title{
TROMBOEMBOLIC EVENTS IN PREGNANT WOMEN AND PUERPERAS: A REVIEW OF THE LITERATURE
}

\begin{abstract}
Venous thromboembolism (VTE) is one of the main causes of obstetric morbimortality in women who are in the pregnancy-puerperal cycle, since the blood hypercoagulability, provided in pregnancy, significantly increases the probability of the onset of this disease, which is associated with some risk factors, often unknown. The objective of this study was to identify the risk factors associated with thromboembolic events in women in the pregnancy-puerperal cycle. A literature review was conducted using the electronic databases PUBMED (Public Medline) and SCIELO (Scientific Electronic Library Online). The following descriptors were used to survey the studies and expand the searches: Portuguese - Risk Factors; Gestation; Pregnancy; Puerperium; Venous Thromboembolism - Risk Factors; Gestation; Pregnancy; Puerperium; Venous Thromboembolism. Sample selection obeyed the following inclusion criteria: complete articles available electronically; be written in the Portuguese language; studies published in the temporal record between 2006 and 2016. Thirty risk factors related to thromboembolic events were identified in the samples from the selected studies, seven of which were evidenced more than once in the studies: age, smoking, obesity, thrombophilia, heart disease, third trimester and prolonged rest. Among the most frequent risk factors that lead pregnant and postpartum women to undergo thromboembolic events, obesity and the age factor greater than 35 years can be highlighted as significant conditions; being the age a risk factor for both antenatal and puerperal. Factors such as smoking, thrombophilia, heart disease, third trimester and prolonged rest were equally relevant for the occurrence of venous thromboembolism.
\end{abstract}

KEYWORDS: Gestation. Pregnancy. Puerperium. Risk Factors. Venous Thromboembolism.

\section{INTRODUÇÃO}

O tromboembolismo é um evento raro e tem sido pouco descrito na literatura (TORRES et al., 2014). Segundo Paiva et al. (2013), os eventos tromboembólicos aumentam a mortalidade por se apresentarem de forma clínica com sintomas escassos, que por muitas vezes, são inespecíficos, o que acabam por dificultar a intervenção imediata. É a terceira causa de mortalidade no mundo, perdendo apenas para infarto agudo do miocárdio e acidente vascular encefálico (FERNANDES et al., 2016).

Segundo a Sociedade Brasileira de Cirurgia Vascular (2012), o tromboembolismo é caracterizado pelo aumento ou distúrbio de coagulação sanguínea nas veias. Ocorre principalmente em veias dos membros inferiores, que ocorrem em cerca de $90 \%$ dos casos, não descartando o acometimento em outros tipos de vasos. Os sinais e sintomas dos eventos tromboembólicos estão restritos a dor e edemas. Esses achados clínicos dificultam o diagnóstico precoce do tromboembolismo. Cabe destacar que as cirurgias de médio e grande portes, infecções graves, idades avançadas, pacientes com anormalidades genéticas no sistema de coagulação, traumatismo, imobilização prolongada como coma e paralisias, infarto agudo do miocárdio e até viagens aéreas longas, assim como a fase final da gestação e o puerpério (pós-parto) estão entre os fatores de risco para o desenvolvimento da trombose venosa profunda. 
De acordo com o Manual Técnico de Gestação de Alto Risco, a promoção da maternidade segura é compromisso do Ministério da Saúde e de todos os profissionais da saúde. Além de garantir o pré-natal e humanizar o atendimento, entre outras ações, é preciso proporcionar atenção especial a uma pequena parcela de mulheres grávidas que são portadoras de doenças que podem se agravar durante a gestação ou que apresentarão problemas que podem ter sido desencadeados nesse período, como o tromboembolismo venoso (BRASIL, 2010).

Por ser uma doença de difícil diagnóstico, os sinais e sintomas do tromboembolismo são facilmente diagnosticados como alterações normais do ciclo gravídico, sendo assim, cabe destacar que deve-se dispensar uma atenção especial de toda equipe multiprofissional que atende às demandas de pacientes gestantes, em busca de um melhor detalhamento da história de vida e de doenças pregressas dessa gestante, para que se possa garantir assistência adequada à saúde dessas pacientes e evitar complicações futuras (ALMEIDA et al., 2015).

Um estudo realizado em 12 pacientes com disfunção ventricular grave destacou que as pacientes ao serem submetidas ao ecocardiograma apresentavam uma fração de ejeção menor que $40 \%$ durante a gestação, destacou que as pacientes estavam evoluindo com complicações clínicas como dispneia, arritmias, acidente vascular encefálico e tromboembolismo, e que quando apresentavam piora progressiva, e foram registrados episódios de paradas cardíacas e até óbito (CUSTÓDIO et al., 2008).

São considerados alguns fatores de risco predisponentes para essa doença que são demonstrados em evidências científicas e, saber identificá-los, é de grande importância para que os profissionais de saúde do Sistema Único de Saúde (SUS) incorporem esses novos conhecimentos na prática, para que a redução da morbidade e mortalidade materna e perinatal possam ser alcançadas (BRASIL, 2010).

Devido ao estado de hipercoagulabilidade que a gestação ocasiona, juntamente com alterações hormonais e fisiológicas, a gestante ou puérpera pode desenvolver trombose venosa profunda que poderá provocar tromboembolismo venoso e diversas outras complicações (BENFATTI et al., 2011).

Os principais fatores de risco para esses fenômenos tromboembólicos na gestação e puerpério são idade maior que 30 ou 35 anos, multiparidade, cesariana (principalmente se de urgência), hipertensão específica da gestação, diabetes gestacional, obesidade, hiperemese gravídica, história pessoal ou familiar de trombose, hemorragia pós-parto, anemia falciforme, lúpus eritematoso sistêmico, infecções ou internações na gravidez, trabalho de parto prematuro, feto morto, gravidez múltipla, cor negra, antecedentes de uso de técnicas de reprodução assistida, trombofilia e tabagismo. Sendo assim, identificar os fatores de risco para trombose venosa profunda nas gestantes e puérperas é fundamental para avaliar a necessidade de profilaxia e diagnóstico (HILLMANN et al., 2015).

O Royal College of Obstetricians \& Gynaecologists divulgou novo guideline para redução de riscos de tromboembolismo venoso (TEV) durante a gravidez e o puerpério. Destacando que o tratamento deve ser realizado com anticoagulante e que a hospitalização pelo tromboembolismo produz um impacto financeiro elevado nos sistemas de saúde. Para a prevenção do mesmo, é necessário reconhecer os fatores de risco considerando fatores de recidiva como tromboembolismo prévio, tromboembolismo por repetição, tromboembolismo venoso na gravidez e no puerpério, tromboembolismo na vigência de uso de anticoncepcionais orais e 
tromboembolismo sem fator desencadeante (ROYAL COLLEGE OF OBSTETRICIANS \& GYNAECOLOHISTS, 2015).

Outra complicação ligada à trombose e relacionada aos fatores estudados que a predispõe, como a gestação e o puerpério, a trombose venosa cerebral (TVC) é uma complicação rara, mas grave. Em termos epidemiológicos a TVC é mais comum em mulheres, entre 20 e 35 anos, e parece não haver predomínio étnico. Geralmente é de início agudo durante a gravidez e na maioria dos casos ocorre no período pós-parto. Quando relacionada ao período pós-parto, a TVC pode ter um início agudo ou mais prolongado. A congestão venosa e o dano ao endotélio vascular, que podem ser secundários ao trabalho de parto e ao período expulsivo, em combinação com o estado de hipercoagulabilidade típico do período pós-parto, poderiam contribuir para o aumento do risco após o nascimento (BISINOTTO et al., 2014).

Desta forma, há de se afirmar que a identificação de fatores de riscos e dos problemas que alteram a qualidade de vida das gestantes de alto risco, estão baseadas em evidências científicas e que são fortemente prevenidas quando observados rigorosamente sinais e sintomas nas consultas pré-natais (ERRICO et al., 2017). Segundo Oliveira e Marques (2016a) o julgamento clínico é fundamental para que se inicie o tratamento profilático por meio de anticoagulantes, o que pode diminuir a taxa de complicações agudas graves e até de óbito dos pacientes acometidos pelo tromboembolismo venoso.

O tratamento do tromboembolismo em gestantes tem sido um dilema médico, pois a prevenção do mesmo com a terapia anticoagulante pode estar relacionada a riscos de hemorragia, teratogenicidade e óbito fetal (ÁVILA, 2010a). O objetivo desse estudo foi realizar uma revisão integrativa de literatura a fim de identificar os fatores de risco associados aos eventos tromboembólicos em mulheres no ciclo gravídico-puerperal.

\section{MATERIAL E MÉTODOS}

A revisão integrativa inicia com um problema de pesquisa, a partir da definição da questão norteadora que neste estudo foi: Quais os fatores que predispõem a ocorrência de trombose venosa na gestação e puerpério? Em seguida, foram definidas as bases de dados para o levantamento dos artigos na literatura, sendo assim, as buscas foram realizadas nas bases de dados da PUBMED (Public Medline) e SCIELO (Scientific Eletronic Library Online), pois ambas disponibilizam o acesso gratuito.

A partir disto, foram definidos os descritores com consulta à base de dados dos Descritores em Ciências da Saúde (DeSC) da Biblioteca Virtual da Saúde em português. Os descritores foram então utilizados para a busca dos artigos, desde que constassem também na da Medical Subject Headings (Mesh), pois tratam-se de palavras-chave de busca internacionalmente padronizadas. Após definição os seguintes descritores e suas combinações em português foram realizadas: Fatores de Risco; Gestação; Gravidez; Puerpério; Tromboembolismo Venoso. Em inglês os descritores pertinentes foram: Risk Factors; Gestation; Pregnancy; Puerperium; Venous Thromboembolism. Ambos os descritores foram combinados entre si nas bases de dados científicas selecionadas para que se possa identificar a literatura publicada no período corte a que se propôs este estudo.

A seleção de amostra obedeceu aos seguintes critérios de inclusão: artigos completos disponíveis eletronicamente e gratuitos, estar escrito no idioma português ou apresentar disponibilidade do estudo traduzido para a língua portuguesa. Foram 
incluídos somente os estudos publicados no período compreendido entre 2006 a 2016.

Como critérios de exclusão foram excluídos os artigos repetidos e os artigos que, ainda que completos, não se relacionassem com o objetivo proposto neste estudo. Inicialmente os artigos foram selecionados pelo título após a combinação de descritores em cada base de dados, aqueles que demonstraram de forma superficial a afinidade com o objetivo do presente estudo, passaram para etapa seguinte com a leitura dos resumos das pesquisas encontradas, descartando os estudos que fugiram da temática proposta nesta revisão. A leitura dos resumos selecionou os artigos que apresentara pertinência com a proposta desta pesquisa, passando para a etapa seguinte com a leitura completa do estudo.

Foram incluídos nesta revisão os artigos completos publicados em português, que retrataram a temática definida na questão norteadora, tendo sido aproveitados os estudos de caso e estudos de análise do discurso sempre disponíveis na íntegra, desde que pudessem contribuir para a síntese do tema estudado. A combinação de descritores identificou um total de 101 artigos, dos quais foram excluídos 89 artigos após a aplicação de filtros corte pela temporalidade e por não atenderem aos critérios de inclusão estabelecidos anteriormente.

As combinações dos descritores foram realizadas igualmente nas duas bases de dados científicas selecionadas, sendo assim, as combinações em português foram: fatores de risco e tromboembolismo venoso e gestação; fatores de risco e tromboembolismo venoso e puerpério; fatores de risco e tromboembolismo venoso. As combinações em inglês foram risk factors and thromboembolism and gestation; risk factors and pregnancy and venous thromboembolismo; e risk factors and puerperium and venous thromboembolism.

Para facilitar a combinação e a busca dos artigos nas bases de dados, optouse pela utilização do conectivo boleano and no Medical Subject Headings Terms (MeSH terms) conforme combinações anteriormente descritas. Os artigos selecionados nas etapas anteriores passaram para a última etapa com a realização de uma leitura minuciosa do conteúdo da pesquisa na íntegra. Nesta etapa, os artigos foram lidos integralmente, sendo excluídos nove estudos devido ao fato de não apresentarem respostas para a questão de pesquisa ou para o objetivo do estudo proposto.

Por conseguinte, o corpus da revisão integrativa só foi possível com as combinações descritas na figura 1, sendo composto por seis artigos, sendo que tanto a análise quanto a síntese dos dados extraídos dos artigos foram realizados de forma descritiva, possibilitando observar, contar, descrever e classificar os dados com o intuito de reunir o conhecimento produzido sobre o tema explorado na revisão, como apresentado na figura abaixo:

\begin{tabular}{|c|c|c|c|c|}
\hline $\begin{array}{l}\text { Trombose } \\
\text { AND } \\
\text { Gestação }\end{array}$ & SCIELO & $\begin{array}{c}49 \\
\text { artigos }\end{array}$ & $\begin{array}{l}15 \text { artigos após } \\
\text { leitura do resumo }\end{array}$ & $\begin{array}{l}4 \text { artigo após } \\
\text { leitura na íntegra }\end{array}$ \\
\hline $\begin{array}{l}\text { AND } \\
\text { Puerpério }\end{array}$ & Pubmed & $\begin{array}{c}52 \\
\text { artigos }\end{array}$ & $\begin{array}{l}12 \text { artigos após } \\
\text { leitura do resumo }\end{array}$ & $\begin{array}{l}2 \text { artigos após } \\
\text { leitura na íntegra }\end{array}$ \\
\hline
\end{tabular}

FIGURA 1 - Demonstrativo do balanço dos artigos analisados para reciclar os principais resultados. Fonte: os autores, (2018). 


\section{RESULTADOS E DISCUSSÃO}

O quadro abaixo apresenta os estudos selecionados para a presente pesquisa de revisão integrativa, obtidas a partir da combinação dos descritores obtidos na Biblioteca Virtual em Saúde e no Medical Subject Headings, sendo possível a seleção de seis artigos dos quais dois foram obtidos na PubMED e quatro na Scielo.

Cabe destacar que os estudos selecionados foram obtidos a partir da combinação e análise por pares, considerando a escassez de estudos da temática publicados no período de 2006 a 2016, estando disponíveis na íntegra e de forma gratuita para os leitores. A baixa disponibilidade de artigos disponíveis para esta pesquisa pode representar uma limitação para este estudo, sugerindo assim, dada a gravidade dos problemas que o tromboembolismo representa para a saúde das gestantes, que novos estudos possam ser realizados sobre a temática.

O Quadro 1 destaca a síntese dos estudos incluídos nesta revisão integrativa de acordo com as bases em que os artigos foram encontrados, os autores, nome do artigo, tipo de estudo e o ano de publicação.

QUADRO 1 - Representação das publicações incluídas na revisão integrativa, com base no título do artigo, base de dados indexada, autores, tipo de estudo e ano de publicação.

\begin{tabular}{|c|c|c|c|c|c|}
\hline Artigo & $\begin{array}{l}\text { Base de } \\
\text { Dados }\end{array}$ & Título Artigo & Autores & Ano & $\begin{array}{l}\text { Tipo de } \\
\text { Estudo }\end{array}$ \\
\hline E1 & PUBMED & $\begin{array}{l}\text { Investigação da trombose } \\
\text { venosa na gravidez. }\end{array}$ & Kalil et al. & 2008 & $\begin{array}{l}\text { Artigo } \\
\text { original }\end{array}$ \\
\hline E2 & SCIELO & $\begin{array}{l}\text { Profilaxia de tromboembolismo } \\
\text { venoso na gestação }\end{array}$ & $\begin{array}{l}\text { Oliveira e } \\
\text { Marques. }\end{array}$ & 2016 & $\begin{array}{l}\text { Artigo de } \\
\text { revisão }\end{array}$ \\
\hline E3 & PUBMED & $\begin{array}{l}\text { Anticoagulação durante } \\
\text { gravidez de mulher portadora } \\
\text { de cardiopatia }\end{array}$ & Ávila & 2010 & $\begin{array}{l}\text { Artigo de } \\
\text { revisão }\end{array}$ \\
\hline E4 & SCIELO & $\begin{array}{l}\text { Trombofilia hereditária: um } \\
\text { caso, várias questões }\end{array}$ & $\begin{array}{l}\text { Lima; } \\
\text { Moreira }\end{array}$ & 2015 & $\begin{array}{l}\text { Relato } \\
\text { de caso }\end{array}$ \\
\hline E5 & SCIELO & $\begin{array}{lr}\text { Associação } & \text { entre } \\
\text { abortamentos } & \text { recorrentes, } \\
\text { perdas fetais, pré-eclâmpsia } \\
\text { grave e } \\
\text { hereditárias e trombofilias } \\
\text { antifosfolípides em mulherpos } \\
\text { do Brasil Central }\end{array}$ & $\begin{array}{l}\text { Figueiró- } \\
\text { Filho e } \\
\text { Oliveira }\end{array}$ & 2007 & $\begin{array}{l}\text { Artigo } \\
\text { original }\end{array}$ \\
\hline E6 & SCIELO & $\begin{array}{l}\text { Resultados gestacionais e } \\
\text { trombofilias em mulheres com } \\
\text { história de óbito fetal de } \\
\text { repetição }\end{array}$ & $\begin{array}{l}\text { Barros et } \\
\text { al. }\end{array}$ & 2014 & $\begin{array}{l}\text { Artigo } \\
\text { original }\end{array}$ \\
\hline
\end{tabular}

Fonte: os autores, (2018). 
No Quadro 2, esta apresentada a síntese dos principais riscos relacionados aos eventos tromboembolíticos relacionados ao estado gravídico e ao puerpério.

QUADRO 2 - Principais fatores de risco relacionados aos eventos tromboembólicos encontrados nos estudos selecionados:

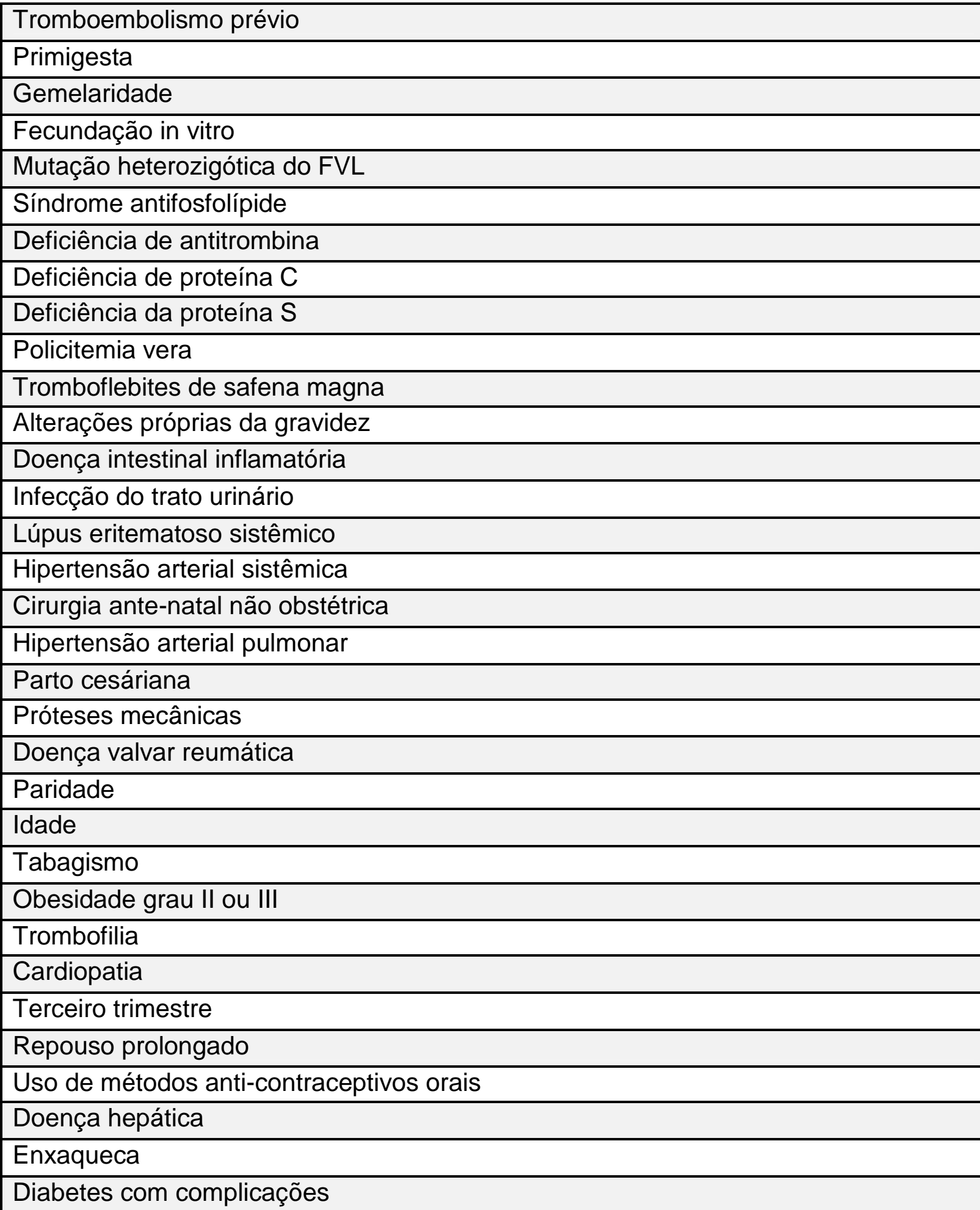

Fonte: os autores, (2018).

Kalil et a.,l (2008) desenvolveram uma pesquisa de investigação sobre trombose venosa na gravidez em um hospital de São Paulo entre janeiro de 2004 e ENCICLOPÉDIA BIOSFERA, Centro Científico Conhecer - Goiânia, v.15 n.28; p.1219 2018 
novembro de 2006. Foram registrados 25.055 nascimentos sendo 21.748 cesarianas $(89 \%), 1.832$ partos normais $(7,5 \%)$ e 857 fórceps (3,5\%). Desse total de parturientes 14.110 eram primíparas $(57,7 \%)$. Cabe ressaltar que partos cesarianos compõem os fatores de riscos para o desenvolvimento de tromboembolismo venoso.

No mesmo estudo, as pacientes com suspeita clínica de trombose venosa profunda foram encaminhadas para atendimento especializado e 42 diagnósticos de trombose foram confirmados. Dessas 42 pacientes com trombose venosa profunda, 32 eram primigestas (76\%), das quais três gemelares e duas por fecundação in vitro, oito secundigestas (19\%) e duas tercigestas (KALIL et al., 2008).

No estudo de Kalil et al., (2008), a idade das pacientes variou entre 21 e 39 anos, duas destas pacientes eram tabagistas ativas. Em quatro pacientes a trombose venosa profunda (TVP) ocorreu no primeiro trimestre da gravidez $(9,5 \%)$; em 11 , no segundo trimestre $(26,2 \%)$; em 27 , no terceiro $(64,3 \%)$, incluindo quatro pós-cesarianas. Houve um caso de tromboembolismo pulmonar no primeiro dia póscesariana em paciente de 37 anos que havia realizado fecundação in vitro, com gravidez gemelar. Ainda nesta pesquisa, 38 pacientes desenvolveram TVP entre a $9^{\circ}$ e a $36^{\circ}$ semana, quatro tromboses $(9,5 \%)$ ocorreram entre o primeiro e o terceiro dia pós-operatório. Não houve TVP em partos normais ou fórceps.

Ainda de acordo com Kalil et al., (2008), os locais mais acometidos foram o membro inferior esquerdo, sendo um total de $23(54,9 \%)$; o território infra patelar apresentou 18 casos (42,8\%), 10 casos em veias poplíteas $(23,8 \%)$, sete nas femorais $(16,6 \%)$, cinco nas ilíacas $(11,9 \%)$ e dois nas safenas magnas $(4,7 \%)$. Em 16 pacientes (38\%) foram apresentados alguns resultados para a etiologia da TVP, sendo que 12 pacientes (28,5\%) tiveram resultados positivos para trombofilia em exames de coagulação; seis pacientes $(14,3 \%)$ apresentaram mutação heterozigótica do FVL; três $(7,1 \%)$ eram portadoras da síndrome antifosfolípide, deficiência de antitrombina, deficiência de proteína $C$ e da proteína $S$, uma paciente cada.

Quatro grávidas não-trombofílicas tiveram como causa determinada de trombose os seguintes fatores: policitemia vera (um caso); viagem longa de avião (um caso); e tromboflebites de safena magna em terço proximal da coxa (dois casos). Os outros 26 casos $(61,9 \%)$ foram atribuídos às alterações próprias da gravidez.. Três pacientes foram excluídas do grupo de trombofilia por não possuírem resultado de exame satisfatório.

De acordo com Oliveira e Marques (2016b), estima-se que de 79 a $89 \%$ das gestantes que morrem por embolia pulmonar apresentam ao menos um fator de risco identificável. O TEV prévio e a presença de trombofilia previamente diagnosticada são duas condições de risco de TEV em gestantes que podem ser reconhecidos antes da gravidez. Conforme estes mesmos autores estudos mencionam que as trombofilias hereditárias são observadas em 20 a $50 \%$ dos casos de ocorrência de TEV na gestação. Nas gestantes com TEV prévio, o risco de recidiva é de 24,8 vezes mais frequente.

Outro fator de risco para o TEV na gestação é a obesidade e esse risco aumenta à medida que o IMC se eleva, o IMC>30 kg/m² é associado ao aumento de 14,9 vezes o risco de EP e TVP. O sobrepeso materno (IMC entre 25 e 29,9 kg/m²) é condição de risco muito comum para o tromboembolismo venoso relacionado à gestação.

Para Oliveira e Marques (2016b), a idade maior que 35 anos é um indicador de risco para a ocorrência de eventos tromboembólicos. Os dados sugerem aumento de risco de duas vezes para mulheres com mais de 35 anos. A 
eventualidade de TEV não aumentou com a idade no período antes do parto; mas mulheres em fase puerperal, entre 35 e 44 anos risco $70 \%$ superior em comparação àquelas entre 25 e 34 anos. Em geral, considera-se a idade de 35 anos ou mais como condição de risco antes e após o nascimento.

Segundo Oliveira e Marques (2016a), os dados relacionados às viagens de longas distâncias e imobilidade em gestantes são limitados, pois são necessários mais estudos de populações não gestantes. As diretrizes relacionadas a cuidados antenatais do Instituto Nacional de Excelência em Saúde e Cuidados (NICE) e as recomendações do RCOG sobre viagens aéreas durante a gestação afirmam que voos com duração superior a quatro horas aumentam o risco de TEV.

Em conformidade com Oliveira e Marques (2016a), a admissão hospitalar durante a gestação é ligada ao aumento de 18 vezes no risco de tromboembolismo venoso em comparação ao risco basal fora do hospital e o risco permanece alto após o puerpério, seis vezes maior, nos 28 dias seguintes o risco é maior no terceiro trimestre de gravidez e em mulheres acima de 35 anos durante a internação hospitalar.

Outras condições para TEV relacionadas à gestação foram citadas como: doença intestinal inflamatória, infecção do trato urinário, lúpus eritematoso sistêmico, cardiopatias, hipertensão arterial sistêmica induzida pela gestação ou pré- eclampsia e cirurgia antenatal não obstétrica (OLIVEIRA; MARQUES, 2016b). Segundo Ávila (2010b), em estudo (E3) realizado com 19 casos de tromboembolismo na gestação, a doença valvar reumática correspondeu a $12 \%(63,1 \%)$ e a seis $(31,6 \%)$ casos distribuídos em cardiopatia congênita e hipertensão arterial pulmonar, respectivamente.

O potencial tromboembólico está centrado, habitualmente, na situação clínica da paciente, mas é agravado pelas particularidades próprias da gravidez, como as reológicas, mecânicas e hormonais, mais proeminentes a partir do segundo trimestre da gravidez e chegando a alcançar o puerpério (ÁVILA, 2010a). Agregam-se a estas outras condições de risco como a idade, paridade, tabagismo, obesidade, repouso prolongado, parto cesárea, que elevam a incidência de fenômeno tromboembólico no ciclo gravídico-puerperal. $\mathrm{O}$ uso de próteses mecânicas ocasionou 0 tromboembolismo e complicações hemorrágicas em 2,0 e 2,5\% dos casos respectivamente (ÁVILA, 2010a).

Segundo o autor acima, constatou-se por meio dos estudos E1, E2, E3, E4, $\mathrm{E} 5$, que dentre os fatores de risco mais frequentes que levam mulheres grávidas e puérperas a sofrerem eventos tromboembólicos, pode-se destacar a obesidade e o fator idade maior que 35 anos como condições significativas; sendo a idade um fator de risco tanto para antenatal como puerperal, seguido por tabagismo, trombofilia, cardiopatias, terceiro trimestre e repouso prolongado.

Outros fatores consideráveis foram encontrados em mulheres como infecção pós-parto, não sendo destacado o sítio de infecção, porém classificada como grave com necessidade de internação urgente em unidade de terapia intensiva (MASCARELLO et al. 2017).

De acordo com Oliveira e Marques (2016b), outras causas para o tromboembolismo venoso são relativas a doenças intestinais inflamatórias, infecção urinária, lúpus eritematoso sistêmico, cardiopatias, hipertensão arterial sistêmica induzida pela gestação ou pré-eclampsia e cirurgia anterior à gestação não obstétrica.

Foi compreendido que a maioria das mulheres sofreu trombose venosa profunda (TVP) no terceiro trimestre entre a $9^{\circ}$ e a $36^{\circ}$ semana. As regiões mais 
acometidas foram o membro inferior esquerdo e o território infra patelar. Nesta revisão o Estudo 1 (E1) de Kalil et al. (2008), apontou que a gestação gemelar, a cesariana e a inseminação artificial também foram fatores predisponentes para a ocorrência de trombose venosa profunda (TVP).

Para Onzi et al., (2007), é possível identificar os fatores de risco em cerca de $80 \%$ das TVP. Porém, um estudo recente sobre o tromboembolismo venoso destacou que esta complicação de saúde é mais frequente na região Sul do Brasil e que isto está relacionado com as baixas temperaturas presentes nestas regiões (OHKI; BELLEN, 2017).

Bisinotto et al., (2014), confirmam que fenômenos tromboembólicos são comuns em mulheres entre 20 e 35 anos, e parece não haver predomínio étnico. A deficiência de antitrobina, proteína $C$ e proteína $S$, fator de Leiden e mutação do gene da protrombina estão presentes em metade dos casos de tromboembolismo na gravidez e no puerpério (HILLMANN et al., 2015).

Destaca-se que muitos eventos tromboembólicos são fatais e ocorrem nos primeiros três meses após o desencadeamento da doença. Sendo assim, é de extrema importância o reconhecimento desses fatores pelos profissionais que irão receber essas mulheres na primeira consulta pré-natal, quando deverá ser instituída a profilaxia antenatal (OLIVEIRA; MARQUES, 2016b).

$\mathrm{Na}$ pesquisa de Figueiró-Filho e Oliveira (2007) sobre a associação entre abortamentos recorrentes, perdas fetais e outras complicações em mulheres grávidas, estes autores concluíram que a identificação de pacientes portadoras de trombofilias é importante para a prevenção de acidentes tromboembólicos. Este mesmo estudo destacou que na gravidez há aumento dos níveis dos fatores de coagulação dessas pacientes.

Em relação a óbitos fetais, Barros et al., (2014a) sobre resultados gestacionais e trombofilias em mulheres com história de óbito fetal e de repetição, foram avaliadas 20 pacientes com histórico de óbito fetal, das quais 3,7\% das pacientes apresentaram fetos natimortos, mesmo aquelas em acompanhamento pelo setor de tromboses e gestação. Com relação à idade, cada paciente tinha em média 30 anos, com uma variação entre 18 e 37 anos. A maior faixa de risco estava no primeiro trimestre. Quanto à paridade, a média ficou entre cinco e oito gestações, variando de três a nove gestações, sendo que cerca de $20 \%$ das pacientes estudadas já haviam apresentado abortamentos anteriormente.

Neste mesmo estudo as complicações obstétricas nas gestações índices ocorreram em 15 das 20 pacientes pesquisadas e foram semelhantes entre aquelas com e sem trombofilias. Dentre as complicações na gestação identificadas na pesquisa, estão a restrição de crescimento fetal intrauterino (25\%), placenta prévia (15\%), índice de líquido amniótico diminuído (20\%), pré-eclâmpsia superajuntada (10\%), sofrimento fetal (5\%), óbito fetal (5\%) e diabetes gestacional (5\%) (BARROS et al., 2014a).

Cabe ressaltar que no estudo de Barros et al.,(2014b) as pacientes com um passado obstétrico comprometido já com histórico de complicações, apresentaram óbitos fetais de repetição. Contudo, os resultados gestacionais foram positivos em cerca de $95 \%$ das pacientes pesquisadas. Entre as pacientes estudadas, $75 \%$ apresentaram complicações obstétricas, sendo que 35\% apresentaram trombofilias e $40 \%$ das pacientes sem trombofilias. A presença de hematomas subcoriônicos ou infartos placentários ocorreu em cinco pacientes (25\%), naquelas com trombofilias.

$\mathrm{Na}$ pesquisa de Lima e Moreira (2015) houve o relato do caso de uma paciente jovem sem antecedentes mórbidos pessoais negando uso de drogas lícitas 
ou ilícitas que desenvolveu um quadro súbito de dispneia, palpitações e dor torácica, procurou a emergência e foi medicada sendo liberada em seguida. O quadro apresentou piora, quando retornou para o hospital, a radiografia pulmonar evidenciava imagens sugestivas, tendo sido internada para tratamento confirmado de tromboembolismo pulmonar extenso. A mesma estava em uso de contracepção com implante progestivo subcutâneo após terminar hipocoagulação terapêutica, já que havia suspendido este método aos 25 anos com desejo de engravidar, desconhecendo casos de tromboembolismo na família.

Sendo assim, destaca-se que mesmo mulheres saudáveis podem desenvolver tromboembolismo venoso, devendo procurar auxílio médico sempre que desejar uma gravidez para que se possa realizar uma investigação de hereditariedade (LIMA; MOREIRA, 2015). Em relação às gestantes, que fazem parte do grupo de risco para o tromboembolismo venoso, observa-se que o grupo de pacientes de médio e alto risco tratados por clínicos receberam mais profilaxia que aqueles manejados por cirurgiões. Em relação à profilaxia para o TEV do total de 78 prontuários avaliados no estudo de Lopes et al., (2017) 70 pacientes receberam anticoagulantes ou compressão pneumática intermitente associado à meia elástica ou deambulação precoce. A profilaxia para eventos tromboembolíticos possui mais eficácia que o tratamento para este tipo de agravamento à saúde dos pacientes independente da condição de vida atual (LOPES et al., 2017).

\section{CONCLUSÃO}

Concluiu-se que o ciclo gravídico-puerperal possui vários fatores de riscos para o desenvolvimento de tromboembolismo venoso.Dentre os fatores de risco mais frequentes que levam mulheres grávidas e puérperas a sofrerem eventos tromboembólicos, podem- se destacar a obesidade e o fator idade maior que 35 anos como condições significativas; sendo a idade um fator de risco tanto para antenatal como puerperal. Seguido por tabagismo, trombofilia, cardiopatias, terceiro trimestre e repouso prolongado.

A maioria das mulheres sofreu algumas das ocorrências tromboembólicas citadas durante o terceiro trimestre entre a nona e trigésima sexta semana. Os locais mais prejudicados foram o membro inferior esquerdo e o território infra-patelar, seguido pelas veias poplíteas e femorais. Outros fatores pertinentes classificados como condições de risco foram: mulheres primigestas, com gestação gemelar; submetidas à fecundação in vitro; pacientes trombofílicas; viagem longa de avião; parto cesariano.

Sugere-se a realização de mais estudos sobre a temática abordada, por considerar a gravidade deste problema e por considerar também que os custos com o tratamento possam estar onerando os planos de saúde e o próprio sistema público de saúde, uma vez que os tratamentos com a prevenção são eficazes e apresentam melhor custo-benefício.

\section{REFERÊNCIAS}

ALMEIDA, K. A; ROCKEN, M. H; RAVELLI, A. P. X. Trombose venosa profunda em gestantes da terceira regional de saúde do Paraná: fatores de risco. Publicação da UEPG Ciências Biológicas e Saúde, Ponta Grossa, v. 21, n.2, p. 145-149, jul./dez, 2015. Disponível em: www.revistas2.uepg.br/index.php/biologica/article/view/8639. Acesso em: 08 de nov $2018 . \quad$ DOI: http://dx.doi.org/10.5212/publicatio\%20uepg.v21i2.8639. 
ÁVILA, W. S. Anticoagulação durante gravidez de mulher portadora de cardiopatia. Jornal Vascular Brasileiro. Porto Alegre, v. 9, n. 1, p. 42-45, 2010a. Disponível em http://www.scielo.br/pdf/jvb/v9n1/0910.pdf. Acesso em 11 de jul. 2017. Epub 23-Abr2010. http://dx.doi.org/10.1590/S1677-54492010005000009.

Anticoagulação durante gravidez de mulher portadora de cardiopatia. Jornal Vascular Brasileiro. Porto Alegre, v. 9, n. 1, p. 42-45, 2010b. Disponível em http://www.scielo.br/pdf/jvb/v9n1/0910.pdf. Acesso em 11 de jul. 2017. Epub 23-Abr2010. http://dx.doi.org/10.1590/S1677-54492010005000009.

BARROS V. I. P. V. L; IGAI, A. M. K; ANDRES, MP, FRANCISCO, R. P. V. F; ZUGAIB. Resultados gestacionais e trombofilias em mulheres com história de óbito fetal de repetição. Revista Brasileira de Ginecologia e Obstetrícia. 2014a; 36(2):50-5. Disponível em: www.scielo.br/pdf/rbgo/v36n2/0100-7203-rbgo-36-0200050.pdf. Acesso em 8 de nov 2018. DOI: http://dx.doi.org/10.1590/S010072032014000200002.

Resultados gestacionais e trombofilias em mulheres com história de óbito fetal de repetição. Revista Brasileira de Ginecologia e Obstetrícia. 2014b; 36(2):50-5. Disponível em: www.scielo.br/pdf/rbgo/v36n2/0100-7203-rbgo-36-0200050.pdf. Acesso em 8 de nov 2018. DOI: http://dx.doi.org/10.1590/S010072032014000200002 .

BENFATTI, R. A; JUNIOR, C. R. M; SILFA, G. V. R; PONTOS, J. C. D. V. Puérpera com trombose de prótese mecânica e estenose supravalvar aórtica adquirida. Brazilian Jounarl of Cardiovascular Surgery, v. 26, no. 2, São José do Rio Preto, apr/jun, $2011 . \quad$ Disponível em: http://www.scielo.br/scielo.php?script=sci_arttext\&pid=S0102-76382011000200022 Acesso em 07 de nov de 2018. DOI: http://dx.doi.org/10.1590/S010276382011000200022 .

BISINOTTO, F. M. B; DEZENA, R. A; ABUD, T. M. V; MARTINS, L. B. Trombose venosa cerebral após raquianestesia: relato de caso. Brazilian Journal of Anesthesiology. Campinas, v. 67, n. 3, p. 305-310, jun. 2014. Disponível em: https://www.sciencedirect.com/science/article/pii/S003470941500029X https://doi.org/10.1016/j.bjan.2014.09.005. Acesso em 11 de jul 2017. DOI: http://dx.doi.org/10.1016/j.bjane.2014.09.015.

BRASIL. Ministério da Saúde. Manual técnico de gestação de alto risco. 5 ed. Brasília: Editora do Ministério da Saúde, 2010.

CUSTÓDIO, M. G; YAMAKAMI, L. Y. S; BORTOLOTTO, M. R. F. L; WAISSMAN, A. L; ZUGAIB, M. Resultados maternos e perinatais em pacientes com disfunção sistólica grave. Revistada da Associação Médica Brasileira [Internet]. 2008 Dec; 54 (6): 500-505. Disponível em: http://www.scielo.br/scielo.php?script=sci_arttext\&pid=S010442302008000600013\&Ing=en Acesso em 07 de nov 2018. DOI: http://dx.doi.org/10.1590/S0104-42302008000600013. 
ERRICO, L. S. P; BICALHO, P. G; OLIVEIRA, T. C. F.L; MARTINS, E. F. O trabalho do enfermeiro no pré-natal de alto risco sob a ótica das necessidades humanas básicas. Acesso em: http://www.scielo.br/pdf/reben/v71s3/pt 0034-7167-reben-71s3-1257.pdf. Acesso em: 7 de nov de 2018. DOI: http://dx.doi.org/10.1590/00347167-2017-0328.

FERNANDES, C. J. C. S; ALVES, JÚNIOR J. L; GAVILANES, F; PRADA, L. F; MORINAGA $L$ et al. Novos anticoagulantes para o tratamento do tromboembolismo venoso. Jornal Brasileiro de Pneumologia [Internet], abr, 2016; 42 (2): 146-154. Disponível em: http://www.scielo.br/scielo.php?script=sci_arttext\&pid=S180637132016000200146\&lng=en. Acesso em 06 de nov 2018. DOI: http://dx.doi.org/10.1590/S1806-37562016042020068.

FIGUEIRÓ-FILHO, EA; OLIVEIRA, VM. Associação entre abortamentos recorrentes, perdas fetais, pré-eclâmpsia grave e trombofilias hereditárias e anticorpos antifosfolípides em mulheres do Brasil Central. Revista Brasileira de Ginecologia e Obstetrícia, 2007; $29 \quad$ (11): 561-7. Disponível em: http://www.scielo.br/pdf/rbgo/v29n11/a03v2911.pdf. Acesso em 8 de nov 2018. DOI: http://dx.doi.org/10.1590/S0100-72032007001100003.

HILLMANN, B. R; STEFFENS, S. M; TRAPANI JUNIOR, A. Trombose de veia renal no puerpério: relato de caso. Revista Brasileira Ginecologia e Obstetrícia. Rio de Janeiro, v. 37 , n. 12, p. 593-597, dez. 2015. Disponível em: http://www.scielo.br/pdf/rbgo/v37n12/0100-7203-rbgo-37-12-00593.pdf Acesso em 11 jul. 2017. DOI http://dx.doi.org/10;1590/S0100-720320150005455.

KALIL, A. J; JOVINO, M. A. C; LIMA, M. A; KALIL, R; MAGLIARI, M. E. R et al. Investigação da trombose venosa na gravidez. Journal Vascular Brasileiro. Porto Alegre, v. 7 , n. 1, p. 28-37, mar. 2008. Disponível em: http://www.scielo.br/pdf/jvb/v7n1/v7n1a06.pdf Acesso em 07 de nov 2018. DOI: http://dx.doi.org/10.1590/S1677-54492008000100006.

LIMA, R. P; MOREIRA, M. Trombofilia hereditária: um caso, várias questões. Revista Portuguesa de Medicina Geral e Familiar, vol. 31, no. 5, Lisboa, out./2015. Disponível em: www.scielo.mec.pt/pdf/rpmgf/v31n5/v31n5a07.pdf Acesso em 07 de nov 2018.

LOPES, B. A. C; TEIXEIRA, I. P; SOUZA, T. D; TAFAREL, J. R. Sabemos prescrever profilaxia de tromboembolismo venoso nos pacientes internados? Jornal Vascular Brasileiro, 2017; 16 (3): 199-204. Disponível em: http://www.scielo.br/pdf/jvb/v16n3/1677-5449-jvb-1677-5449008516.pdf. Acesso em 07 de nov 2018. DOl: http://dx.doi.org/10.1590/1677-5449.008516.

MASCARELLO, K. C; HORTA, B. L; SILVEIRA, M. F. Complicações maternas e cesáreas sem indicação: revisão sistemática e meta-análise. Revista de Saúde Pública, 51: 1 05, 2017. Disponível em: http://www.scielo.br/pdf/rsp/v51/pt_00348910-rsp-S1518-87872017051000389.pdf Acesso em 07 de nov de 2018. DOI: https://doi.org/10.11606/S1518-8787.2017051000389. 
OLIVEIRA, A. L. M. L; MARQUES, M. A. Profilaxia de tromboembolismo venoso na gestação. Jornal Vascular Brasileiro. Porto Alegre, v. 15, n. 4, p. 293-301, dez. 2016a. Disponível em http://www.scielo.br/pdf/jvb/v15n4/1677-5449-jvb-15-4293.pdf. Acesso em 11 de jul 2017. DOI: http://dx.doi.org/10.1590/16775449.006616.

Profilaxia de tromboembolismo venoso na gestação. Jornal Vascular Brasileiro. Porto Alegre, v. 15, n. 4, p. 293-301, dez. 2016b. Disponível em http://www.scielo.br/pdf/jvb/v15n4/1677-5449-jvb-15-4293.pdf. Acesso em 11 de jul 2017. DOl: http://dx.doi.org/10.1590/16775449.006616.

ONZI, R. R; COSTA, L. F; ANGNES, R. F; DOMINGUES, L. A; MORAES, P et al. Jornal Vascular Brasileiro. Acesso em 11 out. 2017. Disponível em: http://www.scielo.br/pdf/jvb/v6n2/v6n2a16.pdf Acesso em 11 de out 2018. DOI: http://dx.doi.org/10.1590/S1677-54492007000200016.

OHKI, A; BELLEN, B. V. A incidência regional do tromboembolismo venoso no Brasil. Jornal Vascular Brasileiro, col. 16, no. 3, Porto Alegre, july/sept, 2017. Disponível em: www.scielo.br/pdf/jvb/v16n3/1677-5449-jvb-16-3-227.pdf.

DOI: http://dx.doi.org/10.1590/1677-5449.000517.

PAIVA, R. A; GHADRAOUI, J; MACHADO, B. B; AMORIM, N. F. G; FISHDICH, H et al. Revista Brasileira Cirurgia Plástica, 2013: 28 (1): 3-9. Disponível em: www.scielo.br/pdf/rbcp/v28n1/03.pdf. Acesso em 07 de nov 2018. DOl: http://dx.doi.org/10.1590/S1983-51752013000100003.

ROYAL COLLEGE OF OBSTETRECIANS \& GYNAECOLOGISTS. Reducing the risk of venous thromboembolism during pregnancy and the puerperium. Green-top Guideline, p. 2-40, no 37a, april, 2015. Disponível em: https://www.rcog.org.uk/globalassets/documents/guidelines/gtg-37a.pdf Acesso em 07 de nov de 2018.

SOCIEDADE BRASILEIRA DE CIRURGIA VASCULAR. Trombose venosa profunda. [online]. 2018. Disponível em: http://www.sbacv.com.br/artigos/medicos/trombosevenosa-profunda. Acesso em: 5 de out. 2018

TORRES, R. A; TORRES, R. B; CASTILHO, A. S. R; HONORATO, R. Trombose de seios venosos em criança com síndrome nefrótica: relato de caso e revisão da literatura. Revista Brasileira de Terapia Intensiva [online]. 2014, vol.26, n.4, pp.430-434. Disponível em: http://www.scielo.br/scielo.php?pid=S0103507X2014000400430\&script=sci_abstract\&tlng=pt Acesso em 08 de nov 2018. DOI: http://dx.doi.org/10.5935/0103-507X.20140066. 